\title{
Wall stress on ascending thoracic aortic aneurysms with bicuspid compared with tricuspid aortic valve
}

Yue Xuan, $\mathrm{PhD},{ }^{\mathrm{a}}$ Zhongjie Wang, $\mathrm{PhD},{ }^{\mathrm{a}}$ Raymond Liu, BS, ${ }^{\mathrm{a}}$ Henrik Haraldsson, $\mathrm{PhD},{ }^{\mathrm{b}}$ Michael D. Hope, MD, ${ }^{\mathrm{b}}$ David A. Saloner, PhD, ${ }^{\mathrm{b}}$ Julius M. Guccione, $\mathrm{PhD},{ }^{\mathrm{a}}$ Liang Ge, $\mathrm{PhD},{ }^{\mathrm{a}}$ and Elaine Tseng, $\mathrm{MD}^{\mathrm{a}}$

\section{ABSTRACT}

Objective: Guidelines for repair of bicuspid aortic valve-associated ascending thoracic aortic aneurysms have been changing, most recently to the same criteria as tricuspid aortic valve-ascending thoracic aortic aneurysms. Rupture/dissection occurs when wall stress exceeds wall strength. Recent studies suggest similar strength of bicuspid aortic valve versus tricuspid aortic valve-ascending thoracic aortic aneurysms; thus, comparative wall stress may better predict dissection in bicuspid aortic valve versus tricuspid aortic valve-ascending thoracic aortic aneurysms. Our aim was to determine whether bicuspid aortic valve-ascending thoracic aortic aneurysms had higher wall stresses than their tricuspid aortic valve counterparts.

Methods: Patients with bicuspid aortic valve- and tricuspid aortic valveascending thoracic aortic aneurysms (bicuspid aortic valve $=17$, tricuspid aortic valve $=19$ ) greater than $4.5 \mathrm{~cm}$ underwent electrocardiogram-gated computed tomography angiography. Patient-specific 3-dimensional geometry was reconstructed and loaded to systemic pressure after accounting for prestress geometry. Finite element analyses were performed using the LS-DYNA solver (LSTC Inc, Livermore, Calif) with user-defined fiber-embedded material model to determine ascending thoracic aortic aneurysm wall stress.

Results: Bicuspid aortic valve-ascending thoracic aortic aneurysms 99th-percentile longitudinal stresses were $280 \mathrm{kPa}$ versus $242 \mathrm{kPa}(P=.028)$ for tricuspid aortic valve-ascending thoracic aortic aneurysms in systole. These stresses did not correlate to diameter for bicuspid aortic valve-ascending thoracic aortic aneurysms $(r=-0.004)$ but had better correlation to tricuspid aortic valve-ascending thoracic aortic aneurysms diameter $(r=0.677)$. Longitudinal stresses on sinotubular junction were significantly higher in bicuspid aortic valve-ascending thoracic aortic aneurysms than in tricuspid aortic valve-ascending thoracic aortic aneurysms (405 vs $329 \mathrm{kPa}, P=.023$ ). Bicuspid aortic valve-ascending thoracic aortic aneurysm 99th-percentile circumferential stresses were $548 \mathrm{kPa}$ versus $462 \mathrm{kPa}(P=.033)$ for tricuspid aortic valve-ascending thoracic aortic aneurysms, which also did not correlate to bicuspid aortic valve-ascending thoracic aortic aneurysm diameter $(r=0.007)$.

Conclusions: Circumferential and longitudinal stresses were greater in bicuspid aortic valve- than tricuspid aortic valve-ascending thoracic aortic aneurysms and were more pronounced in the sinotubular junction. Peak wall stress did not correlate with bicuspid aortic valve-ascending thoracic aortic aneurysm diameter, suggesting diameter alone in this population may be a poor predictor of dissection risk. Our results highlight the need for patient-specific aneurysm wall stress analysis for accurate dissection risk prediction. (J Thorac Cardiovasc Surg 2018;156:492-500)

From the Departments of ${ }^{\mathrm{a}}$ Surgery and ${ }^{\mathrm{b}}$ Radiology, University of California San Francisco and San Francisco Veterans Affairs Medical Centers, San Francisco, Calif. Funded by the National Institutes of Health, R01HL119857-01A1.

Read at the 43rd Annual Meeting of The Western Thoracic Surgical Association, Colorado Springs, Colorado, June 21-24, 2017.

Received for publication June 22, 2017; revisions received Jan 23, 2018; accepted for publication March 5, 2018; available ahead of print April 13, 2018.

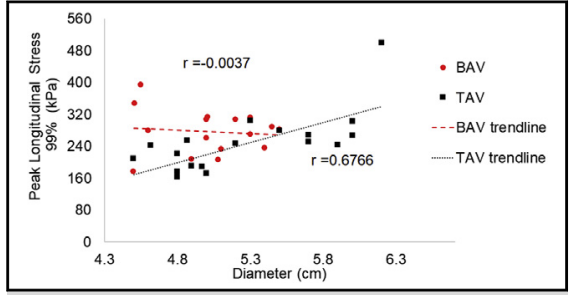

Correlation between $99 \%$ wall stress and maximum aortic diameter

\section{Central Message}

Wall stress was not correlated with BAV-aTAA diameter and would be an important consideration for optimizing the timing of surgical intervention for BAV and likely TAV with less than 5.5-cm aTAA.

\section{Perspective}

We demonstrated that both circumferential and longitudinal stresses were greater in BAV versus TAV-aTAA. Peak wall stresses did not correlate with BAV-aTAA diameter and weakly correlated with TAV-aTAA diameter, suggesting that diameter alone is a poor predictor of aTAA dissection risk and that patient-specific aTAA wall stresses should be considered.

See Editorial Commentary page 501

See Editorial page 471.

Address for reprints: Elaine Tseng, MD, Division of Cardiothoracic Surgery, University of California San Francisco, San Francisco VA Medical Center, 500 Parnassus Ave, Suite 405W, San Francisco, CA 94143 (E-mail: Elaine.Tseng@ucsf.edu). $0022-5223 / \$ 36.00$

Copyright $(\subset) 2018$ by The American Association for Thoracic Surgery https://doi.org/10.1016/j.jtcvs.2018.03.004 

Abbreviations and Acronyms
aTAA $=$ ascending thoracic aortic aneurysm
$\mathrm{BAV}=$ bicuspid aortic valve
$\mathrm{FE}=$ finite element
FEA = finite element analyses
SAVR $=$ surgical aortic valve replacement
STJ = sinotubular junction
$\mathrm{TAV}=$ tricuspid aortic valve

Scanning this QR code will take you to a supplemental video for the article.

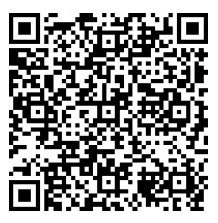

Bicuspid aortic valve (BAV) is the most common congenital aortic valve defect occurring in $0.5 \%$ to $2 \%$ of the general population. However, patients with BAV account for up to $15 \%$ of those presenting with aortic dissection or rupture. ${ }^{1}$ Rupture or dissection of an ascending thoracic aortic aneurysm (aTAA) is a highly lethal condition with a $1 \%$ /hour mortality rate. ${ }^{2}$ To avoid complications of aortic dissection/rupture, the American College of Cardiology and American Heart Association have developed guidelines ${ }^{2-5}$ for elective repair of aTAA, which include consideration of BAV versus tricuspid aortic valve (TAV) phenotype. Previously, these guidelines recommended earlier repair of BAV-aTAA at sizes smaller than recommended for TAV-aTAA, that is, greater than $4.5 \mathrm{~cm}$ versus $5.5 \mathrm{~cm}$, respectively. ${ }^{2}$ These guidelines ${ }^{3}$ recently changed. Operative intervention for BAV-aTAA is now $5.5 \mathrm{~cm}$ or greater for asymptomatic patients and $5.0 \mathrm{~cm}$ or greater for patients with a family history of aortic dissection or aortic growth rate $0.5 \mathrm{~cm} /$ year or greater. Concomitant repair is recommended for BAV-aTAA greater than $4.5 \mathrm{~cm}$ when undergoing surgical aortic valve replacement (SAVR). These guidelines reflect a continually evolving understanding of the biomechanics of aortic dissection.

The aortic wall has a complicated microstructure of collagen and elastin within its 3 layers of intima, media, and adventitia and has the ability to respond to pathophysiologic conditions by remodeling. Dissection/rupture is simply a material failure of the aortic wall and occurs biomechanically when wall stress exceeds wall strength. Studies ${ }^{6-8}$ have suggested that BAV-aTAA tensile strength is equivalent or higher than that of TAV-aTAA. We and others from the International Registry for Aortic Dissection $^{9,10}$ have demonstrated that dissection can occur in a significant proportion of patients with aTAA sizes less than the recommended guidelines. As such, optimal treatment for patients with BAV-aTAA and patients with TAV-aTAA may require elective repair at smaller aTAA sizes in a patient-specific fashion to preclude dissection/ rupture using clinical and biomechanical risk factors. The question remains whether BAV remains a clinical risk factor for dissection from a biomechanics perspective. If BAV has similar or greater wall strength than TAV-aTAA, then comparative wall stress should provide information regarding relative dissection risk of BAV versus TAV.

Wall stress cannot be directly measured; however, stress can be determined computationally. Finite element analysis (FEA) represents a validated technique in computational modeling to investigate mechanical stress in physiologic systems, where stress would otherwise be impossible to measure in vivo. FEA has been widely used to quantify wall stress in arteries. ${ }^{11,12}$ The aim of this study was to compare aTAA wall stress between patients with BAV and patients with TAV using FEA.

\section{MATERIALS AND METHODS}

We performed a retrospective analysis of patients with aTAA from our surgical clinic database at San Francisco Veterans Affairs Medical Center. Inclusion criterion was greater than $4.5 \mathrm{~cm}$ aTAA based on electrocardiogram-gated computed tomography angiography. Exclusion criteria were those with poor image quality resolution or motion artifact on imaging. Patients with previous SAVR or only aortic root dilatation were excluded. There were 36 patients $(\mathrm{BAV}=17$, TAV $=19)$ with aTAA greater than $4.5 \mathrm{~cm}$ and suitable computed tomography angiography for biomechanical evaluation. BAV sub-phenotypes were not differentiated. No patients had a family history of dissection or connective tissue disorder, but 1 patient in each group (BAV and TAV) had a family history of aortic aneurysm. This study was approved by the Committee on Human Research at University of California San Francisco Medical Center and Institutional Review Board at San Francisco Veterans Affairs Medical Center. Table 1 summarizes patient clinical profiles. De-identified images were used to reconstruct 3-dimensional geometry of the aortic root, ascending aorta, and portion of descending thoracic aorta.

\section{Development of Finite Element Model}

A finite element (FE) model for each patient was developed. First, computed tomography scan images were exported as Digital Imaging and Communications in Medicine files and imported into MeVisLab, open source surface reconstruction software (http://www.mevislab.de/home/aboutmevislab) for image segmentation. Next, smooth 3-dimensional surface was constructed and imported into LS-DYNA (LSTC Inc, Livermore, Calif), a commercially available FE software package. LS-DYNA was used for pressure loading simulations and data analysis.

\section{Zero-Pressure Geometry}

CT images used to reconstruct patient-specific 3-dimensional aTAA geometry represented geometry under in vivo physiologic blood pressure conditions and was therefore considered prestressed. FE simulations based on these geometries would load from $0 \mathrm{~mm} \mathrm{Hg}$ to physiologic blood pressure and thus add stress to already prestressed geometry. We and others have demonstrated the importance of accounting for this prestress to accurately determine in vivo wall stress. ${ }^{13}$ We used modified update-Lagrangian 
TABLE 1. Clinical data of bicuspid aortic valve versus tricuspid aortic valve ascending thoracic aortic aneurysms

\begin{tabular}{|c|c|c|c|c|c|}
\hline & \multicolumn{2}{|r|}{ BAV $(n=17)$} & \multicolumn{2}{|c|}{ TAV $(\mathbf{n}=19)$} & $P$ value \\
\hline Aneurysm diameter (cm) & \multicolumn{2}{|r|}{$5.08(4.90-5.30)$} & \multicolumn{2}{|c|}{$5.00(4.84-5.70)$} & .515 \\
\hline \multirow[t]{2}{*}{ Age, y } & \multicolumn{2}{|r|}{$64(61-67)$} & \multicolumn{2}{|c|}{$68(65-75)$} & .133 \\
\hline & No. & $\%$ & No. & $\%$ & \\
\hline \multicolumn{6}{|l|}{ Aortic stenosis } \\
\hline None & 5 & 29.4 & 14 & 73.7 & .392 \\
\hline Mild & 2 & 11.8 & 2 & 10.5 & \\
\hline Moderate & 1 & 5.9 & 0 & 0 & \\
\hline Severe & 9 & 52.9 & 3 & 15.8 & \\
\hline \multicolumn{6}{|l|}{ Aortic insufficiency } \\
\hline None & 6 & 35.3 & 5 & 26.3 & .392 \\
\hline Mild & 8 & 47.1 & 9 & 47.4 & \\
\hline Moderate & 3 & 17.7 & 2 & 10.5 & \\
\hline Severe & 0 & 0 & 3 & 15.8 & \\
\hline
\end{tabular}

Age and diameter are presented as median (25\%-75\% interquartile range). $B A V$, Bicuspid aortic valve; $T A V$, tricuspid aortic valve.

method to calculate prestress. ${ }^{14}$ In this framework, FE geometry is virtually fixed in space, whereas prestress deformation matrix is obtained through an iterative process. Figure 1, $A$ shows representative aTAA FE mesh.

\section{Collagen-Embedded Hyperelastic Material Model}

The aTAA wall was modeled as incompressible hyperelastic material composed of noncollagen matrix reinforced with dispersed collagen fibers. Total strain energy density function for aTAA was derived from the composite of both strain energy density function of ground matrix and that of collagen fibers as:

$$
\Psi(\bar{C})=\Psi_{\text {matrix }}(\bar{C})+\sum_{i=1,2} \Psi_{\text {collageni }}(\bar{C})+\Psi(J)
$$

where $\bar{C}=J^{-\frac{2}{3}} C$ is an isochoric part of the right Cauchy-Green deformation tensor $C$ and $J$ is Jacobian of the deformation gradient, $\Psi(J)$ enforcing the incompressibility of aortic tissue. Ground matrix was assumed to be isotropic and to have neoHookean-like strain energy density function:

$$
\Psi_{\text {matrix }}(\bar{C})=a\left(I_{1}(\bar{C})-3\right)
$$

where $I_{1}(\bar{C})$ is the first invariant of $\bar{C}$ and $a$ is a material constant.

We assumed 2 collagen fibers distributed symmetrically along the circumferential direction (Figure 1, B) with dispersed collage fibers: ${ }^{15}$

$$
\Psi_{\text {collageni }}(\mathrm{C})=\frac{k_{1}}{2 k_{2}}\left[\exp \left(k_{2} \bar{E}_{i}^{2}\right)-1\right], i=1,2
$$

where $\bar{E}_{i}$ is an invariant that reflects the impact of each fiber family deformation on strain energy function ${ }^{15}$ as shown in Figure $1, B ; k_{1}$ and $k_{2}$ are material parameters determined by mechanical testing of the material ${ }^{16}$ (Table 2).
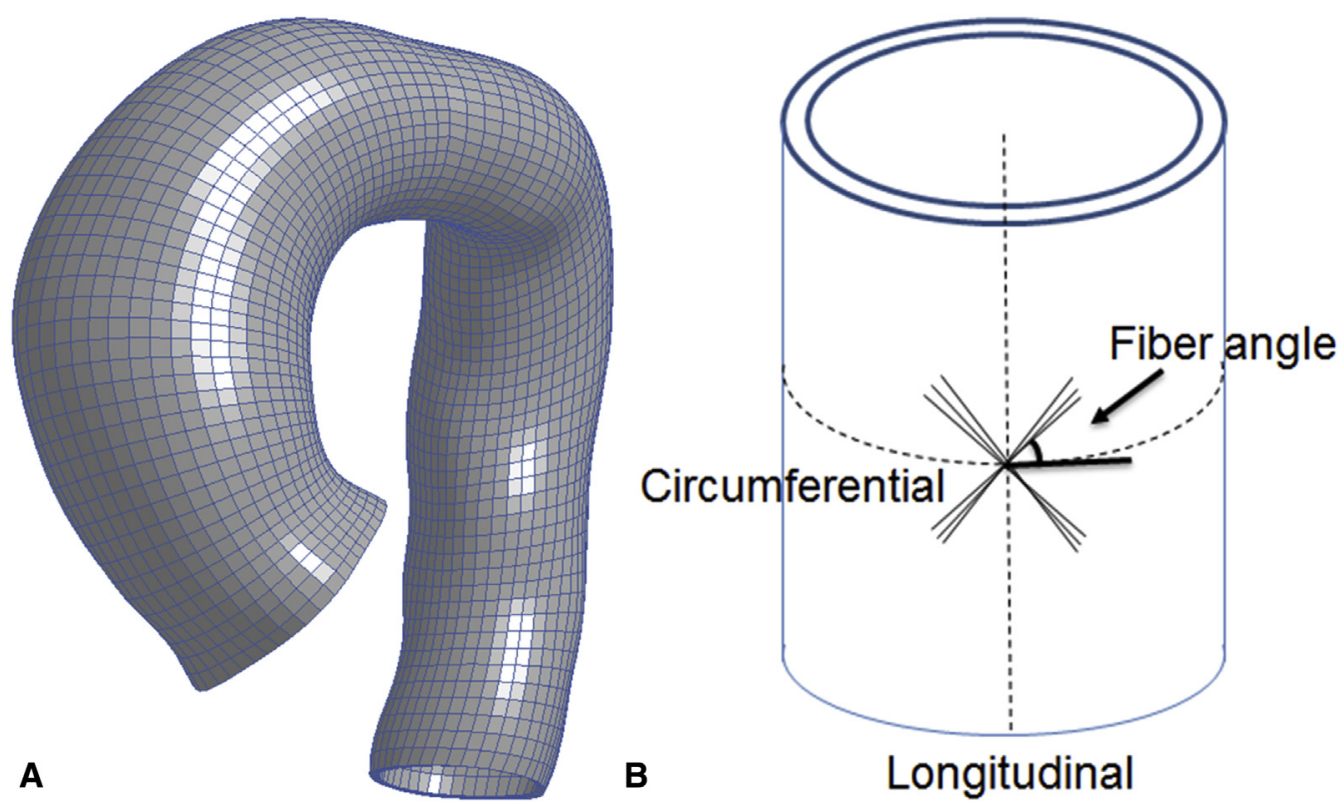

FIGURE 1. A, Representative mesh for FE simulation of aTAA. B, Sketch of fiber angle dispersion with respect to circumferential direction. 
TABLE 2. Material parameters of bicuspid aortic valve versus tricuspid aortic valve ascending thoracic aortic aneurysms

\begin{tabular}{lccc}
\hline Material parameters & $\boldsymbol{k} \boldsymbol{1}$ & $\boldsymbol{k} \boldsymbol{2}$ & Fiber angle (rad) \\
\hline Bicuspid & 66.73 & 17.16 & 0.60 \\
Tricuspid & 84.70 & 9.85 & 0.78 \\
\hline
\end{tabular}

\section{Finite Element Simulation}

FE simulations were performed using LS-DYNA with user-defined material subroutine as described in Equation 1. Reconstructed aTAA wall surface from annulus to descending thoracic aorta was modeled using 3-dimensional brick elements with an average element size of approximately $1.5 \mathrm{~mm}$. All translational motion at the proximal annulus and distal descending thoracic aorta were fixed with rotational freedom. Simulation was performed by applying physiologic arterial pressure loading conditions to aTAA inner lumen. Models were first prestressed to diastolic pressure $(80 \mathrm{~mm} \mathrm{Hg})$. Internal pressure was then ramped up from $80 \mathrm{~mm} \mathrm{Hg}$ to systolic pressure $(120 \mathrm{~mm} \mathrm{Hg})$ over a $100-\mathrm{ms}$ duration, followed by decrease to diastolic pressure over another 100 -ms period. One cardiac cycle of 800-ms duration was then applied. Cardiac cycle was composed of 300 -ms ramp upward to maximum systolic pressure, followed by $500-\mathrm{ms}$ ramp downward to minimum diastolic pressure. Material properties for respective BAV versus TAV-aTAA were used on the basis of our previous biaxial testing. ${ }^{16}$

\section{Statistical Analysis}

The $99^{\text {th }}$-percentile wall stress as previously described ${ }^{17}$ was used for statistical analysis. The 99th-percentile wall stress has been demonstrated
BAV

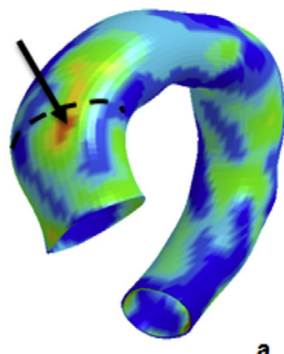

a

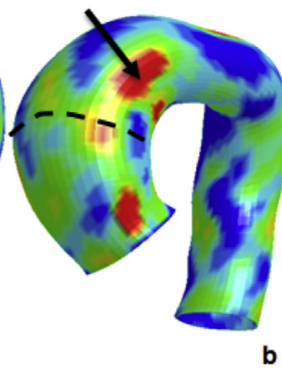

TAV

A
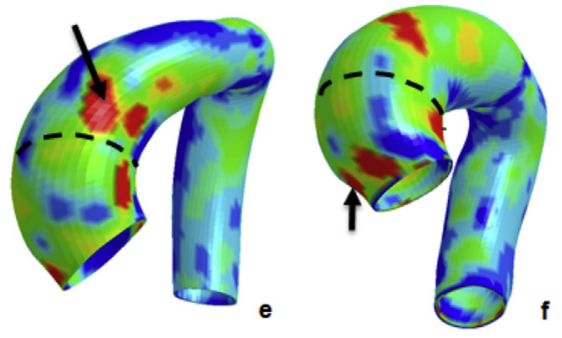

\section{.}
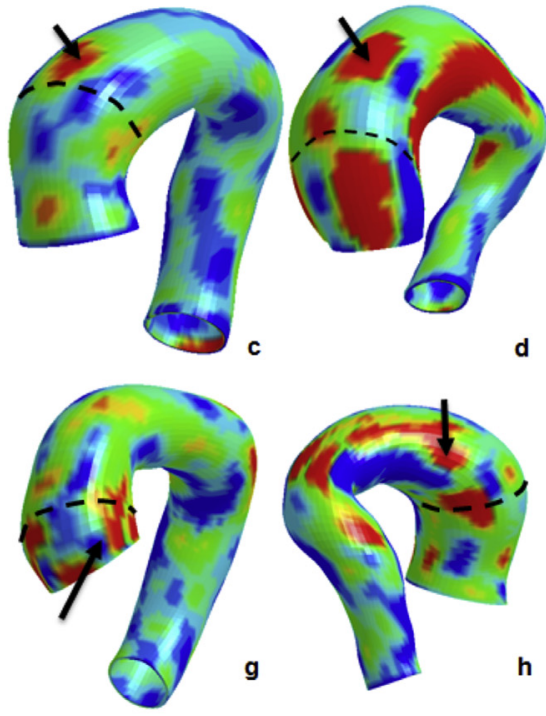

Circumferential Wall Stress

BAV
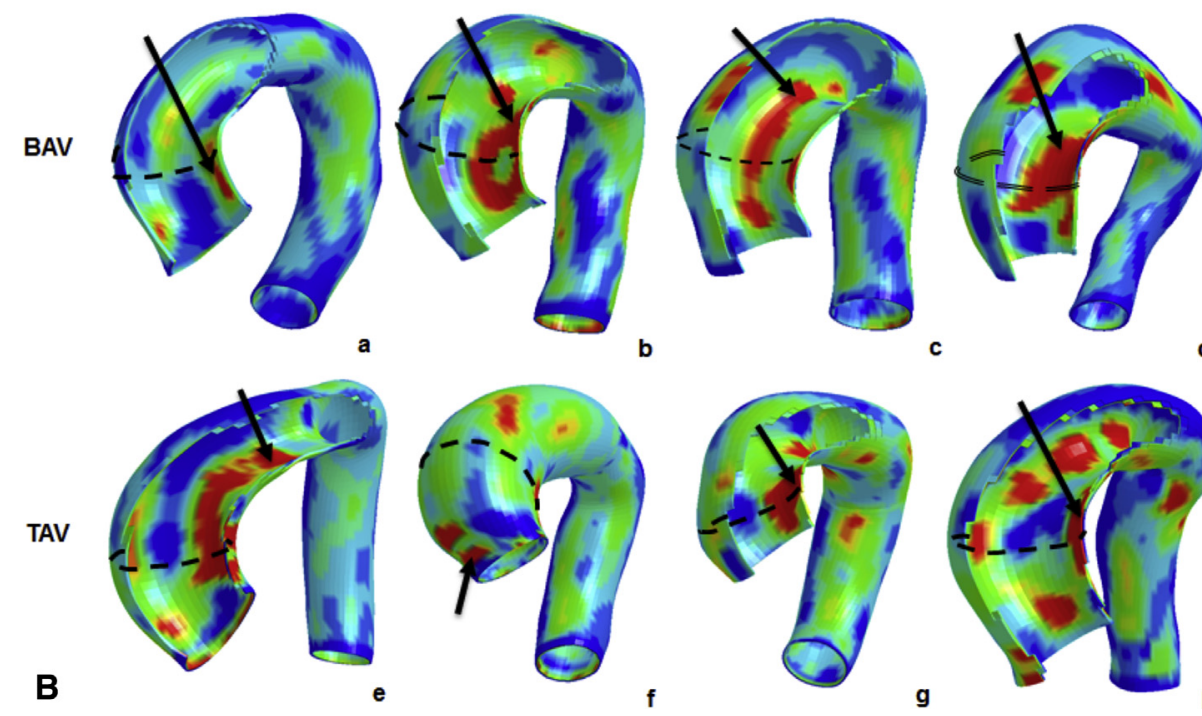

c

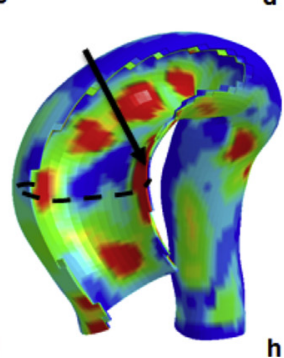

g
$3.000 \mathrm{e}+02$

$2.700 \mathrm{e}+02$

$2.400 \mathrm{e}+02$

$2.100 \mathrm{e}+02$

$1.800 \mathrm{e}+02$

$1.500 \mathrm{e}+02$

$1.200 \mathrm{e}+02$

$9.000 \mathrm{e}+01$

$6.000 \mathrm{e}+01$

$3.000 \mathrm{e}+01$

$0.000 \mathrm{e}+00]$

$5.000 \mathrm{e}+02$
$4.500 \mathrm{e}+02$
$4.000 \mathrm{e}+02$
$3.500 \mathrm{e}+02$
$3.000 \mathrm{e}+02$
$2.500 \mathrm{e}+02$
$2.000 \mathrm{e}+02$
$1.500 \mathrm{e}+02$
$1.000 \mathrm{e}+02$
$5.000 \mathrm{e}+01$
$0.000 \mathrm{e}+00$

FIGURE 2. A, Typical wall stress distribution on BAV $(a-d)$ and TAV-aTAA $(e-h)$ along longitudinal direction. B, Typical wall stress distribution on BAV $(a-d)$ and TAV-aTAA $(e-h)$ along circumferential direction. BAV, Bicuspid aortic valve; TAV, tricuspid aortic valve. 


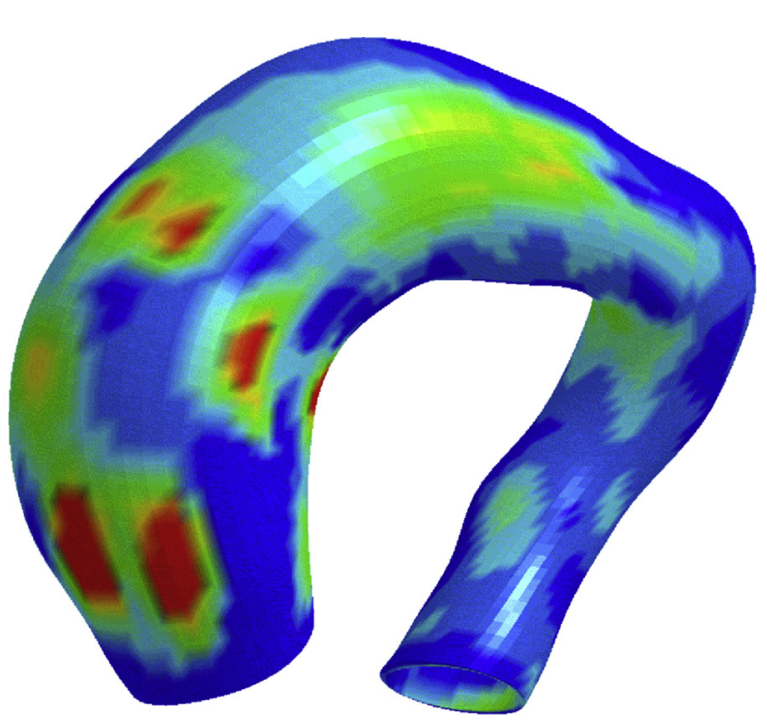

VIDEO 1. Longitudinal stress of BAV-aTAA with systemic pressure loading. Video available at: http://www.jtcvsonline.org/article/S00225223(18)30625-1/fulltext.

to be more reproducible than peak wall stress because it avoids nonphysiologic peak wall stresses that can occur from inhomogeneities in the FE mesh. References to peak wall stresses will hereafter be represented by 99th-percentile wall stress for simplicity. Continuous measurements of aneurysm size, patient age, and wall stress were presented as median and $(25 \%-75 \%)$ interquartile range. Categoric measurements are presented as numbers and percentages. Because the data were not normally distributed, continuous and categoric variables were compared between BAV and TAV cases using the Mann-Whitney $U$ test and KruskalWallis test, respectively. ${ }^{18}$ Spearman rank correlation coefficients were used to determine relationship between aneurysm diameter and wall stress. Statistical analyses were performed using R(R 3.4.0 http://www. r-project.org).

\section{RESULTS}

\section{Patient Demographics}

Patients with BAV and patients with TAV-aTAA had a similar age (64 vs 68 years, $P=.1277$ ), had similar aneurysm sizes (5.08 vs 5, $P=.5152$ ), and had similar incidence of aortic valve disease $(P=.3916)$ (Table 1$)$.

\section{Bicuspid Aortic Valve-Ascending Thoracic Aortic Aneurysm Wall Stress}

BAV-aTAA 99th-percentile longitudinal stresses ${ }^{17}$ were $280 \mathrm{kPa}(236-307 \mathrm{kPa})$ at systolic pressure. There was a trend for highest longitudinal stress to be located on aTAA greater curvature (Figure 2, A, and Video 1). BAVaTAA 99th-percentile circumferential stresses were $548 \mathrm{kPa}(483-595 \mathrm{kPa})$ at systolic pressure. Regions of greatest circumferential stress were located on aTAA lesser curvature (Figure 2, $B$ ). These figures also demonstrate that greatest wall stresses did not localize to the plane of maximum aTAA diameter.
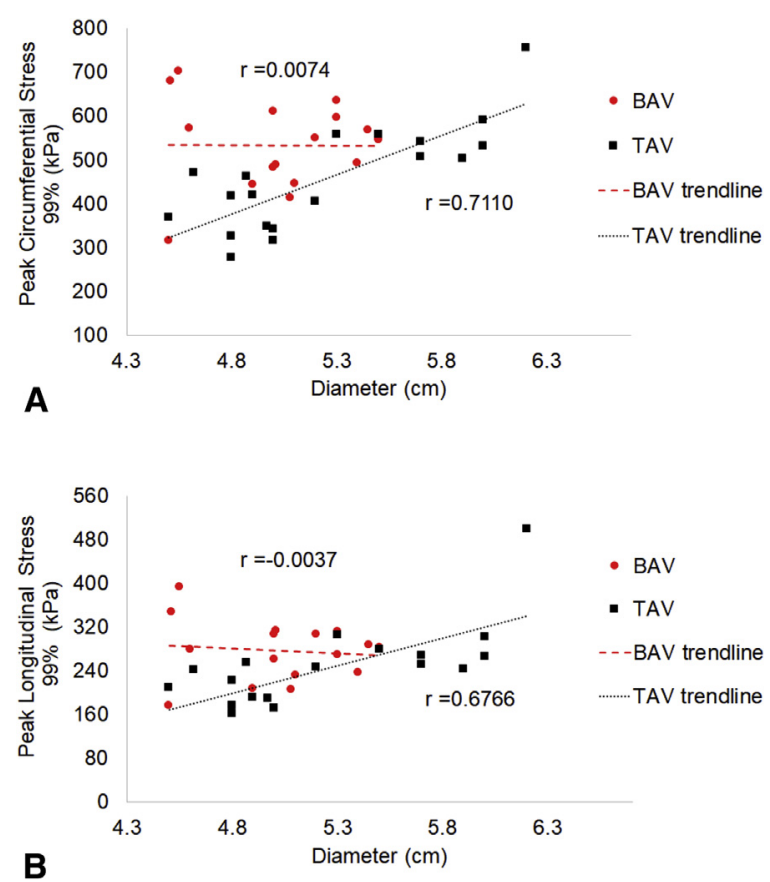

FIGURE 3. A, Relationship between 99th-percentile circumferential stress and aTAA maximum diameter for BAV (circles) and TAV (squares). B, Relationship between 99th-percentile longitudinal stress and aTAA maximum diameter. Correlation between stress and diameter with $r$ is shown as dashed line for BAV and dotted line for TAV. BAV, Bicuspid aortic valve; $T A V$, tricuspid aortic valve.

\section{Tricuspid Aortic Valve-Ascending Thoracic Aortic Aneurysm Wall Stress}

TAV-aTAA 99th-percentile longitudinal stresses were $242 \mathrm{kPa}(189-267 \mathrm{kPa})$ at systolic pressure. No differences were found between greater and lesser curvature regions (Figure 2, A). Peak longitudinal stresses were greater in BAV- than TAV-aTAA $(P=.0275)$. TAV-aTAA 99thpercentile circumferential stresses were $462 \mathrm{kPa}$ (357-536 kPa) at systolic pressure. Similar to BAV-aTAA, regions of highest circumferential stress were located on aTAA lesser curvature (Figure 2, B). Likewise, peak circumferential stresses were greater in BAV- than TAV$\operatorname{aTAA}(P=.033)$.

\section{Ascending Thoracic Aortic Aneurysm Wall Stress Correlation With Diameter}

Maximum aortic diameter and 99th-percentile wall stress were correlated in a linear relationship. For BAV-aTAA, maximum aortic diameter showed no correlation with circumferential or longitudinal 99th-percentile wall stress ( $r=0.0074$ and $r=-0.0037$, respectively) (Figure 3), whereas TAV-aTAA showed better correlation. Correlation between maximum diameter and TAV-aTAA peak wall stress was $r=0.7110$ for circumferential and $r=0.6766$ for longitudinal direction. BAV- and TAV-aTAA 


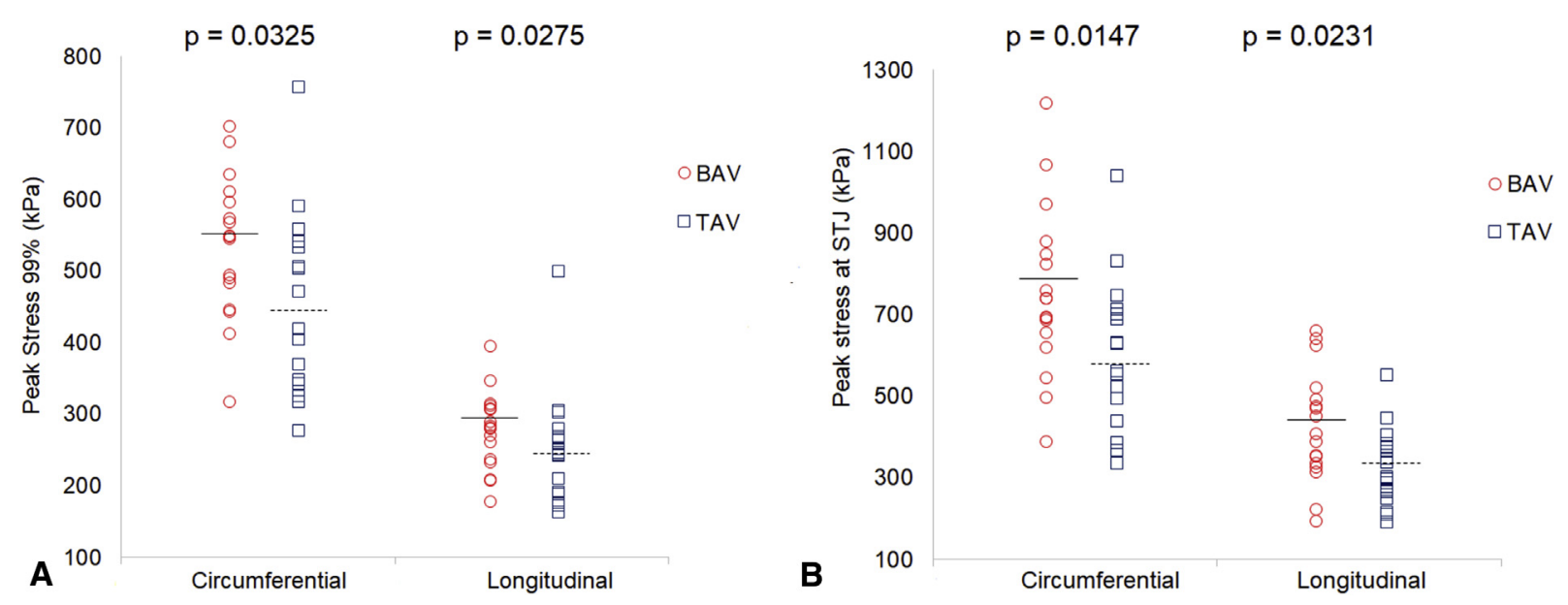

FIGURE 4. Peak wall stress and median values in (A) ascending aorta and (B) STJ of BAV versus TAV-aTAA at systolic pressure with median values in BAV- (solid line) versus TAV-aTAA (dashed line) in systole. BAV, Bicuspid aortic valve; TAV, tricuspid aortic valve; STJ, sinotubular junction.

99th-percentile stresses in circumferential and longitudinal directions in systole are shown in Figure 4, A.

\section{Wall Stress of Sinotubular Junction}

Because the sinotubular junction (STJ) is a wellrecognized region for initial entry tear for acute type A dissection, we analyzed the STJ subregion from above the aortic valve leaflet commissures to $1 \mathrm{~cm}$ distal to the $S T J$. Circumferentially, the STJ peak wall stresses for BAVaTAA were $739 \mathrm{kPa}(654-846 \mathrm{kPa})$ at systolic pressure (Figure $4, B$ ), and those for TAV-aTAA were $560 \mathrm{kPa}$ (498-692 kPa, $P=.015$ ). Longitudinally, STJ peak wall stresses for BAV-aTAA were $405 \mathrm{kPa}(335-489 \mathrm{kPa})$ at systolic pressure compared with those for TAV-aTAA of $329 \mathrm{kPa}(266-377 \mathrm{kPa}, P=.023)$. Correlation between maximum aneurysm diameter and STJ peak stress in circumferential direction was weaker for BAV-aTAA $(r=0.416)$ than for TAV-aTAA $(r=0.600)$, which was also weak. Likewise, correlation between maximum aneurysm diameter and STJ peak stress in longitudinal direction was weaker for BAV-aTAA $(r=0.162)$ than for TAV-aTAA $(r=0.541)$, which also had poor correlation.

STJ greater versus lesser curvature regions were also compared (Table 3). Peak circumferential stresses in BAV-aTAA were significantly larger in the lesser compared with greater curvature of STJ, and peak longitudinal stresses trended toward higher stresses in greater than lesser curvature. On the other hand, peak wall stresses were not significantly different between greater and lesser curvature of STJ for TAV-aTAAs in circumferential or longitudinal directions. By comparing BAV and TAV-aTAAs in greater and lesser curvature STJ subregions, peak circumferential stresses of BAV-aTAAs were significantly greater than that for TAV-aTAAs in both the greater and lesser curvature STJ subregions. In contrast, in the longitudinal direction, no significant differences were found between wall stresses of BAV and TAV-aTAAs in greater or lesser curvature STJ subregions.

\section{DISCUSSION}

\section{Aortic Size and Wall Stress}

American College of Cardiology/American Heart Association guidelines for aTAA elective repair have varied over the years primarily for BAV-aTAA, which decreased from $5.0 \mathrm{~cm}$ or greater in $2006^{4}$ to less than $5 \mathrm{~cm}$ in $2010,{ }^{2}$ and then increased most recently in $2014^{5}$ and $2016^{3}$ to $5.5 \mathrm{~cm}$ or greater, which now matches guidelines for TAV-aTAA of $5.5 \mathrm{~cm}$ or greater unless family history of dissection or growth rate $0.5 \mathrm{~mm} /$ year or greater is present. However, none of these guidelines reflect level A

TABLE 3. Comparison of wall stress in greater and lesser curvature regions of sinotubular junction

\begin{tabular}{lccc}
\hline \multicolumn{1}{c}{ STJ } & BAV & TAV & P value \\
\hline Circumferential-greater curvature & $545(496-612)$ & $432(378-568)$ & .038 \\
Circumferential-lesser curvature & $739(600-863)$ & $521(450-662)$ & .085 \\
$P$ value & .005 & $299(229-368)$ & .008 \\
Longitudinal-greater curvature & $405(249-489)$ & $264(217-331)$ & .076 \\
Longitudinal-lesser curvature & $275(247-331)$ & .358 & .490 \\
$P$ value & .068 & & \\
\hline
\end{tabular}

Stress values are presented as median (interquartile range, $25 \%-75 \%$ ). STJ, Sinotubular junction; $B A V$, bicuspid aortic valve; $T A V$, tricuspid aortic valve. 
evidence, suggesting that better clinical and biomechanical evidence is required than size alone for BAV versus TAV treatment options.

ATAA size with the addition of growth rate and symptoms has served as the basis for timing of elective surgical aTAA repair to avoid the risks of dissection/rupture. However, we and the International Registry for Aortic Dissection have shown acute type A dissection with aortic sizes smaller than the recommended guidelines. ${ }^{10,19} \mathrm{~A}$ biomechanical study also demonstrated that maximum aortic diameter failed to predict rupture/dissection especially for small-sized aTAAs. ${ }^{20}$ Patients with BAV were shown to be more subject to dissection at a smaller size compared with patients with TAV-aTAA, ${ }^{19}$ whereas other studies suggested a low incidence of BAV-aTAA dissection. ${ }^{1,21}$ Although current criteria for BAV-aTAA include a size of $5.5 \mathrm{~cm}$ or greater, high-volume aortic centers recommended early ascending aortic replacement ${ }^{22}$ to reduce the risk of preventable type A dissection for aTAA greater than $5.0 \mathrm{~cm}$. Given the challenges of using size criteria for surgical aTAA repair and conflicting data regarding risks of dissection with BAV versus TAV phenotype, wall stress can provide patient-specific information regarding the risk of dissection and can potentially optimize timing of operative intervention.

In this study, we demonstrated greater peak wall stresses in BAV-aTAA circumferentially than TAV-aTAA in systole. There were no significant differences in longitudinal stresses between BAV and TAV-aTAA cases in systole. However, when we examined the STJ, 1 subregion for intimal tears in type A dissection, there were significantly greater wall stresses in BAV versus TAV-aTAA cases in both circumferential and longitudinal directions. These data suggest that BAV may be at more risk of dissection than TAV-aTAA in that region. We also found that neither circumferential nor longitudinal peak wall stresses correlated with BAV-aTAA maximum diameter. Taken together, these results suggest that diameter may not be a good criterion for evaluation of dissection risk for BAV-aTAA and that patient-specific wall stresses may improve risk stratification. Likewise, although STJ circumferential and longitudinal peak wall stresses showed better correlation with a maximum aTAA diameter for TAV-aTAA than BAVaTAA, overall correlation of wall stress and diameter was still weak. As such, wall stress can be considered an independent factor for dissection than aTAA diameter. Our results also showed that BAV-aTAA of smaller size can have proportionally larger wall stress, suggesting an increased dissection risk when using traditional size criteria. On the other hand, wall stress did not increase with increased diameter for BAV-aTAA cases. Overall, our results suggest the need for patient-specific evaluation of dissection risk based on wall stress. Wall stress is a patient-specific factor driven primarily by patient-specific geometry. Notably, we found the location of greatest wall stress was not found in the plane of maximum aortic diameter. Greatest wall stress occurred by large deformation of a specific area. Thus, our results emphasized the importance of patient-specific wall stress determination to independently evaluate the risk of type A dissection for BAV and TAV-aTAA.

Compared with previous work on aTAA wall stress, our results have some similarity to those from Nathan's group. ${ }^{23}$ They showed that mean 99th-percentile von Mises wall stress in the BAV group was greater than in the TAV group (540 vs $500 \mathrm{kPa}$ ), although without statistical significance, which contrasts with our results. They examined von Mises stress, whereas we studied circumferential versus longitudinal stress. They did not take into account prestress geometry, which we did. Our results showed von Mises stress of $555 \mathrm{kPa}$ for BAV-aTAAs and $450 \mathrm{kPa}$ for TAV-aTAAs with larger aTAA diameters in our study cohort than in their study (5.05 vs $4.0 \mathrm{~cm}$ for BAV, respectively, and $5.25 \mathrm{vs}$ $4.1 \mathrm{~cm}$ for TAV, respectively). Another simulation study of wall stress analysis ${ }^{24}$ showed similar overall peak systolic wall stresses for BAV and TAV-aTAA (average maximum systolic stress 484 vs $471 \mathrm{kPA}$, respectively), for an average aTAA maximum diameter of $5.1 \mathrm{~cm}$ for $\mathrm{BAV}$ and $5.0 \mathrm{~cm}$ for TAV. In that study, they found that aortic size index was suitable for identifying the lowest risk patients for rupture, but unsuitable for distinguishing patients at moderate versus high risk. They suggested that BAVaTAA carried a higher dissection risk than TAV-aTAA despite similar rupture pressures. Our study had similar mean aTAA diameters for BAV and larger diameters for TAV-aTAA than theirs, as well as greater wall stresses based on our patient-specific geometries. We also highlighted that wall stresses in BAV-aTAA could be significantly greater in smaller BAV-aTAA, which is concerning for increased risk of rupture not captured by current guidelines.

\section{Dissection and Wall Strength}

Aortic dissection reflects mechanical failure of the aortic wall, which no longer remains intact at physiologic blood pressure to contain the body's blood circulation. Aortic dissection occurs when aortic wall stress exceeds the wall strength of the intima layer. Previous work ${ }^{6}$ demonstrated greater aTAA wall strength along the circumferential compared with longitudinal direction. These results suggest that the initial failure and intimal tear would begin transversely and propagate along the circumferential spiral. ${ }^{10}$ Transverse tears often occur in acute type A dissection where the initial tear is situated within the first few centimeters of ascending aorta. ${ }^{25}$ When we analyzed the STJ subregion, peak stress along longitudinal direction was greater than that for overall ascending aorta for both BAV (405 vs $280 \mathrm{kPa}$, respectively) and TAV (329 vs $242 \mathrm{kPa}$, respectively), supporting that location for initiating tears. Lower 
STJ wall stress was seen along greater than lesser curvature for BAV-aTAA in the circumferential direction. However, there was a trend toward higher stress in the STJ greater curvature in BAV than TAV-aTAA along the longitudinal direction, which requires a larger patient population for further study. ${ }^{25}$

If patient-specific peak wall stresses remain far below mean tensile strength at physiologic and hypertensive blood pressures, then the risk of dissection should remain low and the aTAA not likely to rupture. Given recent data on failure strength of both BAV- and TAV-aTAAs, patient-specific wall stress analyses can assist clinically in determining timing for elective surgical aTAA repair to prevent risk of dissection, by examining less than $5.5-\mathrm{cm}$ aTAA with peak stresses of concern that approach the tensile strength.

Conflicting data have been reported regarding BAV versus TAV wall strength. Phillippi and colleagues ${ }^{26}$ showed greater wall tensile strength of BAV versus TAVaTAA in both circumferential and longitudinal directions ${ }^{7}$ despite uniform collagen distribution in both. Forsell and colleagues $^{7}$ showed that BAV-aTAA wall strength was 2 times greater than TAV-aTAA with identical collagen orientation. BAV had greater collagen stiffness but equivalent elastin stiffness as TAV-aTAA to account for the overall greater wall strength. In contrast, Pham and colleagues ${ }^{8}$ demonstrated that failure mechanics between BAV and TAV-aTAA were equivalent, and BAV was stiffer than TAV-aTAA, had less elastin, and was thinner. Histologically, studies ${ }^{27}$ have demonstrated accumulation of mucoid material, elastin fragmentation, and change of smooth muscle cell orientation in BAV-aTAA compared with TAVaTAA. Highly aligned elastin and collagen fibers and reduced immature collagen were observed in BAV-aTAA compared with TAV-aTAA. ${ }^{26}$ Clearly, additional work in the field of strength mechanics between BAV and TAVaTAA will be required; however, to date, none have suggested weaker BAV compared with TAV-aTAA wall strength. As such, patient-specific wall stress plays an important role in distinguishing risk of dissection for BAV versus TAV-aTAA.

\section{Influence of Wall Shear Stress}

Wall stress by FEA in this study represents the stress due to blood pressure on aTAA wall. Wall shear stress by blood flow, on the other hand, is orders of magnitude smaller than wall $\operatorname{stress}^{28}$ and represents the stress seen by endothelial cells of intimal layer based on blood flow. One postulate for BAV-aTAA formation is hydrodynamic, based on abnormal flow pattern through BAV leading to helical flow patterns and BAV-aTAA eccentric morphology. ${ }^{28,29}$ Wall shear stress from abnormal blood flow was hypothesized to predispose to aneurysm development, whereas hemodynamics and wall stress acted synergistically to initiate the intimal defect by inducing disruption of aortic wall layers in which biomechanical differences could magnify those effects.

\section{Study Limitations}

One study limitation was the inability to use patientspecific material properties, which may potentially influence results. Determination of in vivo patient-specific material properties requires measurement of in vivo aortic wall motion with costly and time-consuming magnetic resonance imaging with cine displacement encoded imaging with stimulated echoes ${ }^{30}$ (DENSE) and was therefore outside the scope of this study. However, we did use separate material properties for calculating in vivo stress for BAV and TAV-aTAA, which were obtained from mechanical stretch testing to determine averaged material properties for BAV and TAV-aTAA. ${ }^{16}$ Our group is presently quantifying differences in calculated stresses with the use of averaged versus patient-specific material properties in a small subset of patients with surgical aneurysm to determine the impact of material properties on wall stresses. Another limitation was that aTAA regions were assumed to be homogeneous for each patient. However, there is again conflicting evidence regarding the differences in wall thickness between BAV and TAV-aTAA, with one study that showed BAV-aTAA was thinner, ${ }^{31}$ whereas another study showed BAV-aTAA had equivalent thickness as TAV-aTAA. Further information of localized thickness with advances in imaging technique would improve the risk evaluation for dissection. Boundary conditions were fixed for rigid body motion with rotational freedom of the aortic annulus proximally and descending thoracic aorta distally. Anatomically, the ligamentum arteriosum provides restraint that can affect stress results and has particular impact in entry tears for type B aortic dissection. In this study, we did not determine the insertion point of the ligamentum but did include the descending thoracic aorta with a fixed distal end to minimize errors from applying boundary conditions too proximally in the arch. Our model did not include the left ventricle, and thus fixation at the annulus was the most appropriate boundary condition for the current model. Additional factors not modeled that may affect wall stress analysis included passive support from structures in the mediastinum, such as the pulmonary artery, and were beyond the scope of the present work. Last, heterogeneity in stenosis versus regurgitation among our BAV versus TAV population can affect wall shear stresses between the 2 groups. However, wall shear stress is orders of magnitude less than wall stress based on blood pressure. ${ }^{28}$ Wall shear stress caused by blood flow along the intima and affecting endothelial cell lining was beyond the scope of the current study but may help understanding of growth and remodeling of BAV-aTAA based on flow eccentricities in the future. Our study population was limited to men because of the veteran population and requires further study in women. Future studies will be 
performed that examine the influence of valve disease, stenosis versus regurgitation, in combination with valve phenotype, BAV versus $\mathrm{TAV}$, and size on wall stress in aTAAs, but will require a much larger study population for statistical analyses.

\section{CONCLUSIONS}

We determined patient-specific wall stresses in patients with aTAA with BAV versus TAV. Correlation between peak wall stress and aneurysm diameter was found to be weak, especially for BAV-aTAAs, thus highlighting the need for patient-specific aneurysm wall stress analysis to evaluate aortic dissection risk and optimize timing of operative intervention.

\section{Conflict of Interest Statement}

Authors have nothing to disclose with regard to commercial support.

\section{References}

1. Davies RR, Kaple RK, Mandapati D, Gallo A, Botta DM, Elefteriades JA, et al. Natural history of ascending aortic aneurysms in the setting of an unreplaced bicuspid aortic valve. Ann Thorac Surg. 2007;83:1338-44.

2. Hiratzka LF, Bakris GL, Beckman JA, Bersin RM, Carr VF, Casey DE, et al. 2010 ACCF/AHA/AATS/ACR/ASA/SCA/SCAI/SIR/STS/SVM guidelines for the diagnosis and management of patients with thoracic aortic disease. J Am Coll Cardiol. 2010;55:e27-129.

3. Hiratzka LF, Creager MA, Isselbacher EM, Svensson LG, Nishimura RA, Bonow RO, et al. Surgery for aortic dilatation in patients with bicuspid aortic valves: a statement of clarification from the American College of Cardiology/ American Heart Association task force on clinical practice guidelines. J Am Coll Cardiol. 2016;67:724-31.

4. Bonow RO, Carabello BA, Chatterjee K, de Leon AC, Faxon DP, Freed MD, et al. ACC/AHA 2006 guidelines for the management of patients with valvular heart disease: a report of the American College of Cardiology/American Heart Association task force on practice guidelines (writing committee to revise the 1998 guidelines for the management of patients with valvular heart disease) developed in collaboration with the Society of Cardiovascular Anesthesiologists endorsed by the Society for Cardiovascular Angiography and Interventions and the Society of Thoracic Surgeons. J Am Coll Cardiol. 2006;48:e1-148.

5. Nishimura RA, Otto CM, Bonow RO, Carabello BA, Erwin JP III, Guyton RA, et al. 2014 AHA/ACC guideline for the management of patients with valvular heart disease: a report of the American College of Cardiology/American Heart Association task force on practice guidelines. Circulation. 2014;129:e521-643.

6. Pichamuthu JE, Phillippi JA, Cleary DA, Chew DW, Hempel J, Vorp DA, et al. Differential tensile strength and collagen composition in ascending aortic aneurysms by aortic valve phenotype. Ann Thorac Surg. 2013;96:2147-54.

7. Forsell C, Björck HM, Eriksson P, Franco-Cereceda A, Gasser TC. Biomechanical properties of the thoracic aneurysmal wall: differences between bicuspid aortic valve and tricuspid aortic valve patients. Ann Thorac Surg. 2014;98:65-71.

8. Pham T, Martin C, Elefteriades J, Sun W. Biomechanical characterization of ascending aortic aneurysm with concomitant bicuspid aortic valve and bovine aortic arch. Acta Biomater. 2013;9:7927-36.

9. Di Eusanio M, Trimarchi S, Patel HJ, Hutchison S, Suzuki T, Peterson MD, et al. Clinical presentation, management, and short-term outcome of patients with type A acute dissection complicated by mesenteric malperfusion: observations from the international registry of Acute Aortic Dissection. J Thorac Cardiovasc Surg. 2013;145:385-90

10. Jaussaud N, Chitsaz S, Meadows A, Wintermark M, Cambronero N, Azadani AN, et al. Acute type A aortic dissection intimal tears by 64-slice computed tomography: a role for endovascular stent-grafting? J Cardiovasc Surg (Torino). 2013;54:373-81

11. Natsume K, Shiiya N, Takehara Y, Sugiyama M, Satoh H, Yamashita K, et al. Characterizing saccular aortic arch aneurysms from the geometry-flow dynamics relationship. J Thorac Cardiovasc Surg. 2017;153:1413-20.e1.

12. Wang Z, Wood NB, Xu XY. A viscoelastic fluid-structure interaction model for carotid arteries under pulsatile flow. Int J Numer Methods Biomed Eng. 2015;31: e02709.

13. Wisneski AD, Mookhoek A, Chitsaz S, Hope MD, Guccione JM, Ge L, et al. Patient-specific finite element analysis of ascending thoracic aortic aneurysm. $J$ Heart Valve Dis. 2014;23:765.

14. Gee MW, Förster C, Wall WA. A computational strategy for prestressing patientspecific biomechanical problems under finite deformation. Int J Numer Methods Biomed Eng. 2010;26:52-72.

15. Gasser TC, Ogden RW, Holzapfel GA. Hyperelastic modelling of arterial layers with distributed collagen fibre orientations. J $R$ Soc Interface. 2006; 3:15-35.

16. Azadani AN, Chitsaz S, Mannion A, Mookhoek A, Wisneski A, Guccione JM, et al. Biomechanical properties of human ascending thoracic aortic aneurysms. Ann Thorac Surg. 2013;96:50-8.

17. Speelman L, Bosboom EMH, Schurink GWH, Hellenthal FAMVI, Buth J, Breeuwer M, et al. Patient-specific AAA wall stress analysis: 99-percentile versus peak stress. Eur J Vasc Endovasc Surg. 2008;36:668-76.

18. Linhartová K, Beránek V, Sefrna F, Hanisová I, Sterbáková G, Pesková M. Aortic stenosis severity is not a risk factor for poststenotic dilatation of the ascending aorta. Circ J. 2007; 71:84-8.

19. Fedak PW, Verma S, David TE, Leask RL, Weisel RD, Butany J. Clinical and pathophysiological implications of a bicuspid aortic valve. Circulation. 2002; 106:900-4.

20. McGloughlin TM, Doyle BJ. New approaches to abdominal aortic aneurysm rupture risk assessment. Arterioscler Thromb Vasc Biol. 2010;30:1687-94.

21. Michelena HI, Khanna AD, Mahoney D, Margaryan E, Topilsky Y, Suri RM, et al. Incidence of aortic complications in patients with bicuspid aortic valves. JAMA. 2011;306:1104-12.

22. Wojnarski CM, Svensson LG, Roselli EE, Idrees JJ, Lowry AM, Ehrlinger J, et al. Aortic dissection in patients with bicuspid aortic valve-associated aneurysms. Ann Thorac Surg. 2015;100:1666-74.

23. Nathan DP, Xu C, Plappert T, Desjardins B, Gorman JH, Bavaria JE, et al. Increased ascending aortic wall stress in patients with bicuspid aortic valves. Ann Thorac Surg. 2011;92:1384-9.

24. Martin C, Sun W, Elefteriades J. Patient-specific finite element analysis of ascending aorta aneurysms. Am J Physiol Heart Circ Physiol. 2015;308: H1306-16.

25. Ge L, Tseng EE. Invited commentary. Ann Thorac Surg. 2013;96:2154.

26. Phillippi JA, Green BR, Eskay MA, Kotlarczyk MP, Hill MR, Robertson AM, et al. Mechanism of aortic medial matrix remodeling is distinct in patients with bicuspid aortic valve. J Thorac Cardiovasc Surg. 2014; 147:1056-64.

27. de Sa M, Moshkovitz Y, Butany J, David TE. Histologic abnormalities of the ascending aorta and pulmonary trunk in patients with bicuspid aortic valve disease: clinical relevance to the ross procedure. J Thorac Cardiovasc Surg. 1999; 118:588-96.

28. Pasta S, Rinaudo A, Luca A, Pilato M, Scardulla C, Gleason TG, et al. Difference in hemodynamic and wall stress of ascending thoracic aortic aneurysms with bicuspid and tricuspid aortic valve. J Biomech. 2013;46:1729-38.

29. Verma S, Siu SC. Aortic dilatation in patients with bicuspid aortic valve. $N$ Engl J Med. 2014;370:1920-9.

30. Krishnan K, Ge L, Haraldsson H, Hope MD, Saloner DA, Guccione JM, et al. Ascending thoracic aortic aneurysm wall stress analysis using patient-specific finite element modeling of in vivo magnetic resonance imaging. Interact Cardiovasc Thorac Surg. 2015;21:471-80.

31. Choudhury N, Bouchot O, Rouleau L, Tremblay D, Cartier R, Butany J, et al. Local mechanical and structural properties of healthy and diseased human ascending aorta tissue. Cardiovasc Pathol. 2009;18:83-91.

Key Words: aneurysm, bicuspid aortic valve, wall stress 